\title{
CANAL SURFACES WHOSE CENTER CURVE IS THE CURVE IN THE LIGTHLIKE CONE $Q^{2}$
}

\author{
ALI UÇUM* \\ Department of Mathematics, Kırıkkale University, Kırıkkale-Turkey \\ *Corresponding author: aliucum05@gmail.com \\ Received Aug. 18, 2021
}

\begin{abstract}
AвstRACT. In this paper, we obtain the parametrization of the canal surfaces whose center curves are the curves on the lightlike cone $Q^{2}$ in $\mathbb{E}_{1}^{3}$. The parametrization of the canal surface is expressed according to the asymptotic orthonormal frame given in [9]. Then the parallel surface of this surface is studied. Also we define the notion of the associated canal surface. Lastly we give the geometric properties of these surfaces such that Weingarten surface and linear Weingarten surface.
\end{abstract}

2010 Mathematics Subject Classification. 53B30; 53C50; 53A35.

Key words and phrases. canal surfaces; tubular surfaces; Weingarten surface; lightlike cone.

\section{INTRODUCTION}

Canal surfaces was firstly investigated by Monge in 1850. A canal surface is defined as a surface formed as the envelope of a family of spheres whose centers lie on a space curve $C(t)$ with radius $r(t)$. If the radius $r(t)$ is constant, then the canal surface is called as pipe surface or tubular surface. Canal surfaces play an essential role in descriptive geometry, because in case of an orthographic projection its contour curve can be drawn as the envelope of circles. In technical area canal surfaces can be used for blending surfaces smoothly. Canal surface is useful to represent various objects e.g. pipe, hose, rope or intestine of a body. Moreover, canal surface is an important instrument in surface modelling for CAD/CAM such as tubular surfaces, torus and Dupin cyclides [5].

DOI: $10.28924 / A P J M / 8-19$

(2021 Asia Pacific Journal of Mathematics 
Canal surfaces and tubular surfaces have been studied by many researchers. In [3], [4], [5], [6], the authors study canal surfaces and tubular surfaces in Euclidean 3-space, Minkowski 3-space, Galilean and Pseudo Galilean spaces. Lately, in [11], the authors consider the new approach to canal surfaces. Also in [2] and [7], the authors study canal surfaces with quaternions.

In [8] and [9], the author defines the Frenet equations of the curves on the sphere $S^{2}$, hyperbolic sphere $H^{2}$ and lightlike cone.

In this paper, we obtain the parametrization of the canal surfaces whose center curves are the curves on the lightlike cone $Q^{2}$ in $\mathbb{E}_{1}^{3}$. The parametrization of the canal surface is expressed according to the cone frame given in [9]. Then the parallel surface of this surface is studied. Also we define the notion of the associated canal surface. Lastly we give the geometric properties of these surfaces such that Weingarten surface and linear Weingarten surface.

\section{Preliminaries}

The Minkowski space $\mathbb{E}_{1}^{3}$ is the Euclidean 3-space $\mathbb{E}^{3}$ equipped with indefinite flat metric given by

$$
d s^{2}=-d x_{1}^{2}+d x_{2}^{2}+d x_{3}^{2},
$$

where $\left(x_{1}, x_{2}, x_{3}\right)$ is a rectangular coordinate system of $\mathbb{E}_{1}^{3}$. We denote by $\langle$,$\rangle the inner product$ on $\mathbb{E}_{1}^{3}$.

Let $m$ be a fixed point and $r>0$ be a constant. The pseudo-Riemannian sphere is defined by

$$
S_{1}^{2}(m, r)=\left\{u \in \mathbb{E}_{1}^{3}:\langle u-m, u-m\rangle=r^{2}\right\}
$$

the pseudo-Riemannian hyperbolic space is defined by

$$
H^{2}(m, r)=\left\{u \in \mathbb{E}_{1}^{3}:\langle u-m, u-m\rangle=-r^{2}\right\}
$$

the pseudo-Riemannian lightlike cone is defined by

$$
Q^{2}(m)=\left\{u \in \mathbb{E}_{1}^{3}:\langle u-m, u-m\rangle=0\right\} .
$$

For a curve $x(s)$ in the lightlike cone $Q^{2} \subset \mathbb{E}_{1}^{3}$, we choose $\{x(s), \alpha(s), y(s)\}$ forming a Pseudo orthonormal basis of $\mathbb{E}_{1}^{3}$. Then the Frenet formulas of $x(s)$ can be written as

$$
x^{\prime}(s)=\alpha(s), \quad \alpha^{\prime}(s)=\kappa(s) x(s)-y(s), \quad y^{\prime}(s)=-\kappa(s) \alpha(s)
$$

where $\langle x(s), x(s)\rangle=\langle y(s), y(s)\rangle=\langle x(s), \alpha(s)\rangle=\langle y(s), \alpha(s)\rangle=0$ and $\langle x(s), y(s)\rangle=$ $\langle\alpha(s), \alpha(s)\rangle=1$. 
Here, the function $\kappa(s)$ is called the cone curvature function (or curvature) of $x(s)$ and the frame $\{x(s), \alpha(s), y(s)\}$ is called the asymptotic orthonormal frame along the curve $x(s)$ $([9])$.

\section{Canal surfaces with the curve in the ligthlike cone $Q^{2}$}

In this section, we consider the canal surfaces with the curves in the ligthlike cone $Q^{2}$.

Theorem 3.1. Let $x(s)$ be a curve in the lightlike cone $Q^{2}$ and be the center curve of a canal surface ( $i$ ) obtained from the sphere $S_{1}^{2}(r)$. Then the parametrization of the canal surface can be as following

$$
M(s, t)=(1+a(s, t)) x(s)-r(s) r_{s}(s) \alpha(s)+\left(\frac{r^{2}(s)\left(1-r_{s}^{2}(s)\right)}{2 a(s, t)}\right) y(s)
$$

and the parametrization of the tubular surface can be as following

$$
M(s, t)=(1+a(s, t)) x(s)+\frac{r^{2}}{2 a(s, t)} y(s)
$$

(ii) obtained from the hyperbolic space $H^{2}(r)$. Then the parametrization of the canal surface can be as following

$$
M(s, t)=(1+a(s, t)) x(s)+r(s) r_{s}(s) \alpha(s)-\left(\frac{r^{2}(s)\left(1+r_{s}^{2}(s)\right)}{2 a(s, t)}\right) y(s)
$$

and the parametrization of the tubular surface can be as following

$$
M(s, t)=(1+a(s, t)) x(s)-\frac{r^{2}}{2 a(s, t)} y(s)
$$

(iii) obtained from the ligthlike cone $Q^{2}$. Then the parametrization of the canal surface(tubular surface) can be as following

$$
M_{1}(s, t)=(1+a(s, t)) x(s)
$$

or

$$
M_{2}(s, t)=x(s)+a(s, t) y(s)
$$

where $a(s, t)$ is a function of $s$ and $t$, but not only $s$.

Remark 3.2. It is clear that the surfaces

$$
M_{1}(s, t)=(1+a(s, t)) x(s)
$$

or

$$
M_{2}(s, t)=x(s)+a(s, t) y(s)
$$

are degenerate surfaces. 
The following theorem can be seen in [9]. However, we give the theorem by changing the statements of the theorem.

Theorem 3.3. Let $\kappa$ be a real number. Then $x(s)$ is a curve in the lightlike cone $Q^{2}$ with arc-length parameter s and curvature $\kappa$ satisfying

(i) $\kappa>0$ if and only if $x(s)$ can be parameterized by

$$
x=\cosh (\sqrt{2 \kappa} s) V_{1}+\sinh (\sqrt{2 \kappa} s) V_{2}+V_{3}
$$

where $V_{1}, V_{2}, V_{3}$ are mutually orthogonal vectors satisfying the following equations

$$
\left\langle V_{1}, V_{1}\right\rangle=-\left\langle V_{2}, V_{2}\right\rangle=-\left\langle V_{3}, V_{3}\right\rangle=-\frac{1}{2 \kappa} .
$$

(ii) $\kappa<0$ if and only if $x(s)$ can be parameterized by

$$
x=\cos (\sqrt{-2 \kappa} s) V_{1}+\sin (\sqrt{-2 \kappa} s) V_{2}+V_{3}
$$

where $V_{1}, V_{2}, V_{3}$ are mutually orthogonal vectors satisfying the following equations

$$
\left\langle V_{1}, V_{1}\right\rangle=\left\langle V_{2}, V_{2}\right\rangle=-\left\langle V_{3}, V_{3}\right\rangle=-\frac{1}{2 \kappa}
$$

(iii) $\kappa=0$ if and only if $x(s)$ can be parameterized by

$$
x=s^{2} V_{1}+s V_{2}+V_{3}
$$

where

$$
\begin{gathered}
\left\langle V_{1}, V_{1}\right\rangle=\left\langle V_{1}, V_{2}\right\rangle=\left\langle V_{2}, V_{3}\right\rangle=\left\langle V_{3}, V_{3}\right\rangle=0, \\
\left\langle V_{2}, V_{2}\right\rangle=1 \quad \text { and } \quad\left\langle V_{1}, V_{3}\right\rangle=-\frac{1}{2} .
\end{gathered}
$$

Example 1. Let us take $\kappa=1$ in $(i)$ of Theorem 3.3. Then we can choose

$$
V_{1}=\left(\frac{1}{\sqrt{2}}, 0,0\right), \quad V_{2}=\left(0, \frac{1}{\sqrt{2}}, 0\right), \quad V_{3}=\left(0,0, \frac{1}{\sqrt{2}}\right),
$$

which implies that

$$
\begin{aligned}
& x=\left(\frac{\cosh (\sqrt{2} s)}{\sqrt{2}}, \frac{\sinh (\sqrt{2} s)}{\sqrt{2}}, \frac{1}{\sqrt{2}}\right), \\
& \alpha=(\sinh (\sqrt{2} s), \cosh (\sqrt{2} s), 0), \\
& y=\left(-\frac{\cosh (\sqrt{2} s)}{\sqrt{2}},-\frac{\sinh (\sqrt{2} s)}{\sqrt{2}}, \frac{1}{\sqrt{2}}\right) .
\end{aligned}
$$

Now let us give the canal surfaces in Figure 1. 


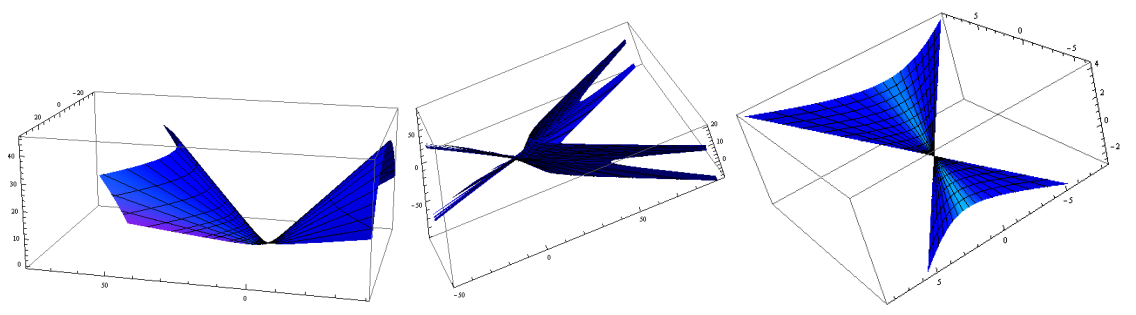

FIgURE 1. The canal surface for $r=2, a=t^{2}$ in $(i)$ of Theorem 3.1 (left), $r=s, a=t$ in (ii) of Theorem 3.1(middle) and $a=t$ in $M_{1}$ of (iii) of Theorem 3.1(right).

Example 2. Let us take $\kappa=-1$ in (ii) of Theorem 3.3. Then we can choose

$$
V_{1}=\left(0, \frac{1}{\sqrt{2}}, 0\right), \quad V_{2}=\left(0,0, \frac{1}{\sqrt{2}}\right), \quad V_{3}=\left(\frac{1}{\sqrt{2}}, 0,0\right)
$$

which implies that

$$
\begin{aligned}
& x=\left(\frac{1}{\sqrt{2}}, \frac{\cos (\sqrt{2} s)}{\sqrt{2}}, \frac{\sin (\sqrt{2} s)}{\sqrt{2}}\right), \\
& \alpha=(0,-\sin (\sqrt{2} s), \cos (\sqrt{2} s)), \\
& y=\left(-\frac{1}{\sqrt{2}}, \frac{\cos (\sqrt{2} s)}{\sqrt{2}}, \frac{\sin (\sqrt{2} s)}{\sqrt{2}}\right) .
\end{aligned}
$$

Now let us give the canal surfaces in Figure 2.

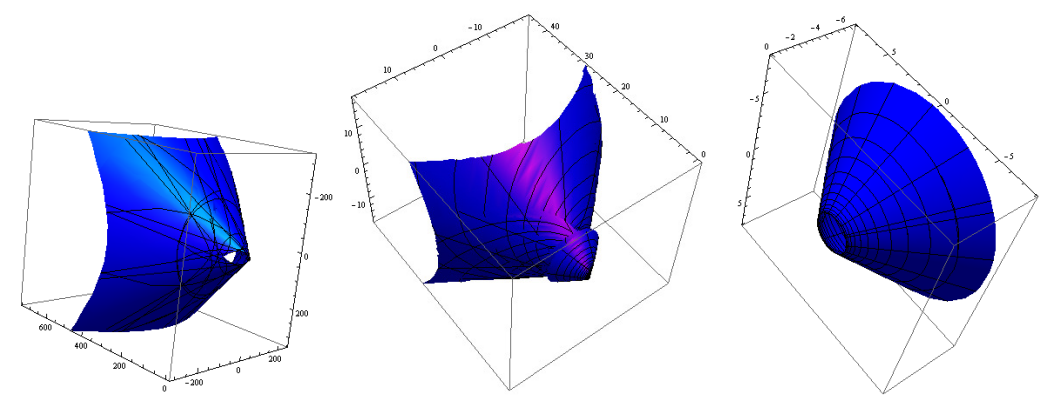

Figure 2. The canal surface for $r=s^{2}, a=\cosh t$ in (i) of Theorem 3.1 (left), $r=s$, $a=e^{t}$ in (ii) of Theorem 3.1 (middle) and $a=\cosh t$ in $M_{2}$ of (iii) of Theorem 3.1(right).

Example 3. Let us take $\kappa=0$ in (iii) of Theorem 3.3. Then we can choose

$$
V_{1}=\left(\frac{1}{4}, \frac{1}{4}, 0\right), \quad V_{2}=(0,0,1), \quad V_{3}=(1,-1,0)
$$


which implies that

$$
\begin{aligned}
x & =\left(\frac{s^{2}}{4}+1, \frac{s^{2}}{4}-1, s\right) \\
\alpha & =\left(\frac{s}{2}, \frac{s}{2}, 1\right) \\
y & =\left(-\frac{1}{2},-\frac{1}{2}, 0\right) .
\end{aligned}
$$

Now let us give the canal surfaces in Figure 3.

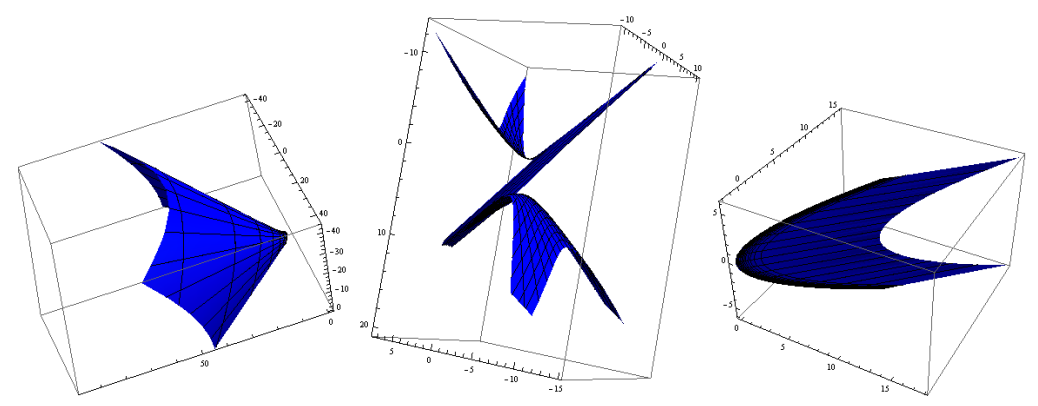

FIgURE 3. The canal surface for $r=2, a=\cosh t$ in $(i)$ of Theorem 3.1 (left), $r=2$, $a=t \cosh (\sqrt{2} s)$ in (ii) of Theorem 3.1(middle) and $a=\ln t$ in $M_{2}$ of (iii) of Theorem 3.1(right).

4. Tubular surface WhOse Center CuRve is the CuRVe in the LigTHliKe CONE $Q^{2}$

In this section we consider the tubular surface whose center curve is the curve in $Q^{2}$.

4.1. Tubular surface obtained from the sphere $S_{1}^{2}(r)$. In this subsection, we consider the tubular surface obtained from the sphere $S_{1}^{2}(r)$, given in theorem 3.1. We assume that $a(s, t)=$ $a(t)$. So we give the parametrization of the tubular surface as

$$
\psi(s, t)=(1+a(t)) x(s)+\frac{r^{2}}{2 a(t)} y(s)
$$

where $a(t)$ is nonconstant function. From (4.1), we find

$$
\begin{aligned}
& \psi_{s}=\left(1+a-\frac{r^{2} \kappa}{2 a}\right) \alpha \\
& \psi_{t}=a^{\prime} x-\frac{r^{2} a^{\prime}}{2 a^{2}} y
\end{aligned}
$$


where $a^{\prime}$ is the derivative of $a$ with respect to $t$. We can find the components of first fundemental form as follows

$$
\begin{aligned}
& g_{11}=\left\langle\psi_{s}, \psi_{s}\right\rangle=\left(1+a-\frac{r^{2} \kappa}{2 a}\right)^{2}, \quad g_{12}=\left\langle\psi_{s}, \psi_{t}\right\rangle=0, \\
& g_{22}=\left\langle\psi_{t}, \psi_{t}\right\rangle=-\frac{r^{2}\left(a^{\prime}\right)^{2}}{a^{2}} .
\end{aligned}
$$

Then $g_{11} g_{22}-\left(g_{12}\right)^{2}=-\frac{r^{2}\left(a^{\prime}\right)^{2}}{a^{2}}\left(1+a-\frac{r^{2} \kappa}{2 a}\right)^{2}$. We assume that

$$
1+a-\frac{r^{2} \kappa}{2 a} \neq 0
$$

for the regularity of the surface $\psi$.

Now we will give an orthonormal basis on $\psi(s, t)$.

$$
\begin{aligned}
e_{1} & =\frac{1}{\left\|\psi_{s}\right\|} \psi_{s}=\varepsilon_{1} \alpha, \\
e_{2} & =\frac{1}{\left\|\psi_{t}\right\|} \psi_{t}=\varepsilon_{2} \varepsilon_{3}\left(\frac{a}{r} x-\frac{r}{2 a} y\right),
\end{aligned}
$$

where $\varepsilon_{1}=\operatorname{sgn}\left(1+a-\frac{r^{2} \kappa}{2 a}\right), \varepsilon_{2}=\operatorname{sgn}(a)$ and $\varepsilon_{3}=\operatorname{sgn}\left(a^{\prime}\right)$. Here $\left\{e_{1}, e_{2}\right\}$ is an orthonormal frame field on $\psi(s, t)$ with signature $(+,-)$. Set

$$
e_{3}=\varepsilon_{2} \frac{a}{r} x+\varepsilon_{2} \frac{r}{2 a} y
$$

where $e_{3}$ is a normal vector field to $\psi(s, t) \cdot\left\{e_{1}, e_{2}, e_{3}\right\}$ is an orthonormal basis on $\psi(s, t)$. Then we obtain

$$
\begin{aligned}
D_{e_{1}} e_{1} & =\frac{2 a}{2 a+2 a^{2}-r^{2} \kappa}(\kappa x-y), \\
D_{e_{1}} e_{2} & =\frac{\varepsilon_{1} \varepsilon_{2} \varepsilon_{3}\left(2 a^{2}+r^{2} \kappa\right)}{r\left(2 a+2 a^{2}-r^{2} \kappa\right)} \alpha, \\
D_{e_{2}} e_{2} & =\frac{a}{r^{2}} x+\frac{1}{2 a} y .
\end{aligned}
$$

The components of the second fundamental form $h$ are calculated as follows

$$
\begin{aligned}
& h_{11}=\left\langle D_{e_{1}} e_{1}, e_{3}\right\rangle=\frac{-\varepsilon_{2}\left(2 a^{2}-r^{2} \kappa\right)}{r\left(2 a+2 a^{2}-r^{2} \kappa\right)}, \\
& h_{12}=\left\langle D_{e_{1}} e_{2}, e_{3}\right\rangle=0 \quad \text { and } \quad h_{22}=\left\langle D_{e_{2}} e_{2}, e_{3}\right\rangle=\frac{\varepsilon_{2}}{r} .
\end{aligned}
$$

Theorem 4.1. The mean curvature $H$ of $\psi(s, t)$ is obtained as

$$
H=\frac{1}{2}\left(h_{11}-h_{22}\right)=\frac{-\varepsilon_{2}\left(a+2 a^{2}-r^{2} \kappa\right)}{r\left(2 a+2 a^{2}-r^{2} \kappa\right)} .
$$


Theorem 4.2. The Gauss curvature $K$ of $\psi(s, t)$ is obtained as

$$
K=-\left(h_{11} h_{22}-\left(h_{12}\right)^{2}\right)=\frac{2 a^{2}-r^{2} \kappa}{r^{2}\left(2 a+2 a^{2}-r^{2} \kappa\right)} .
$$

A surface is called Weingarten surface if there exist a non-trivial function $\Psi(K, H)$ such that $\Psi(K, H)=K_{s} H_{t}-K_{t} H_{s}=0$ for the Gauss curvature $K$ and mean curvature $H$ of the surface. Here subscripts denote partial derivatives. Also we a surface is called as a linear Weingarten surface if there exist real numbers $a, b, c \in \mathbb{R} \backslash\{0\}$ such that the linear combination $a K+b H=c$ is satisfied $([10])$.

From (4.2) and (4.3), we have

$$
K_{s}=\frac{-2 a \kappa^{\prime}}{\left(2 a+2 a^{2}-r^{2} \kappa\right)^{2}}, \quad K_{t}=\frac{2\left(2 a^{2}+r^{2} \kappa\right) a^{\prime}}{r^{2}\left(2 a+2 a^{2}-r^{2} \kappa\right)^{2}}
$$

and

$$
H_{s}=\frac{\varepsilon_{2} r \kappa^{\prime} a}{\left(2 a+2 a^{2}-r^{2} \kappa\right)^{2}}, \quad H_{t}=\frac{-\varepsilon_{2}\left(2 a^{2}+r^{2} \kappa\right) a^{\prime}}{r\left(2 a+2 a^{2}-r^{2} \kappa\right)^{2}} .
$$

Thus it can be easily seen that $\Psi(K, H)=K_{s} H_{t}-K_{t} H_{s}=0$. So we can give the following theorems.

Theorem 4.3. The surface $\psi(s, t)$ is a Weingarten surface.

Theorem 4.4. Let $K$ and $H$ be the Gauss curvature and mean curvature of the surface $\psi(s, t)$. Then there exists the following relation between $K$ and $H$ :

$$
-r^{2} K-2 \varepsilon_{2} r H=1
$$

where $r$ is a positive real number.

From above theorem, we get the following corollary.

Corollary 4.5. The surface $\psi(s, t)$ is a linear Weingarten surface.

Definition 4.6. The parallel surface of the surface $X(s, t)$ defined by

$$
X^{*}(s, t)=X(s, t)+\mu U(s, t)
$$

where

$$
U(s, t)=\frac{X_{s} \times X_{t}}{\left\|X_{s} \times X_{t}\right\|}
$$

is the unit normal vector of the surface $X(s, t)$ and $\mu \in \mathbb{R}$. 
Now we will define the parallel surface $\psi^{*}(s, t)$ of the surface $\psi(s, t)$ as follows

$$
\begin{aligned}
\psi^{*}(s, t) & =\psi(s, t)+\mu e_{3} \\
& =\left(1+a+\varepsilon_{2} \mu \frac{a}{r}\right) x+\left(\frac{r^{2}}{2 a}+\varepsilon_{2} \mu \frac{r}{2 a}\right) y,
\end{aligned}
$$

where $r+\varepsilon_{2} \mu \neq 0$. From (4.4), we find

$$
\begin{aligned}
& \psi_{s}^{*}=\frac{2 r a+2 a^{2}\left(r+\varepsilon_{2} \mu\right)-r^{2}\left(r+\varepsilon_{2} \mu\right) \kappa}{2 r a} \alpha, \\
& \psi_{t}^{*}=\left(a^{\prime}+\varepsilon_{2} \mu \frac{a^{\prime}}{r}\right) x-\left(\frac{r^{2} a^{\prime}}{2 a^{2}}+\varepsilon_{2} \mu \frac{r a^{\prime}}{2 a^{2}}\right) y .
\end{aligned}
$$

We can find the components of first fundemental form as follows

$$
\begin{aligned}
& g_{11}^{*}=\left\langle\psi_{s}^{*}, \psi_{s}^{*}\right\rangle=\frac{\left(2 r a+2 a^{2}\left(r+\varepsilon_{2} \mu\right)-r^{2}\left(r+\varepsilon_{2} \mu\right) \kappa\right)^{2}}{4 r^{2} a^{2}}, \\
& g_{12}^{*}=\left\langle\psi_{s}^{*}, \psi_{t}^{*}\right\rangle=0, \quad g_{22}=\left\langle\psi_{t}, \psi_{t}\right\rangle=-\frac{\left(r+\varepsilon_{2} \mu\right)^{2}\left(a^{\prime}\right)^{2}}{a^{2}} .
\end{aligned}
$$

Theorem 4.7. Let $\psi^{*}(s, t)$ be the parallel surface surface of $\psi(s, t)$. Then $\psi^{*}(s, t)$ has a singular point at $\psi\left(s_{0}, t_{0}\right)$ if and only if

$$
2 r a\left(t_{0}\right)+2 a\left(t_{0}\right)^{2}\left(r+\varepsilon_{2} \mu\right)-r^{2}\left(r+\varepsilon_{2} \mu\right) \kappa\left(s_{0}\right)=0 .
$$

So we assume that $2 r a+2 a^{2}\left(r+\varepsilon_{2} \mu\right)-r^{2}\left(r+\varepsilon_{2} \mu\right) \kappa \neq 0$ for all $\psi(s, t)$. Now we will give an orthonormal basis on $\psi^{*}(s, t)$.

$$
\begin{aligned}
e_{1}^{*} & =\frac{1}{\left\|\psi_{s}^{*}\right\|} \psi_{s}^{*}=\varepsilon_{2} \varepsilon_{4} \alpha, \\
e_{2}^{*} & =\frac{1}{\left\|\psi_{t}^{*}\right\|} \psi_{t}^{*}=\varepsilon_{2} \varepsilon_{3} \varepsilon_{5}\left(\frac{a}{r} x-\frac{r}{2 a} y\right),
\end{aligned}
$$

where $\varepsilon_{4}=\operatorname{sgn}\left(2 r a+2 a^{2}\left(r+\varepsilon_{2} \mu\right)-r^{2}\left(r+\varepsilon_{2} \mu\right) \kappa\right)$ and $\varepsilon_{5}=\operatorname{sgn}\left(r+\varepsilon_{2} \mu\right)$. Here $\left\{e_{1}^{*}, e_{2}^{*}\right\}$ is an orthonormal frame field on $\psi^{*}(s, t)$. Set

$$
e_{3}^{*}=\varepsilon_{2} \varepsilon_{3} \varepsilon_{5}\left(\frac{a}{r} x+\frac{r}{2 a} y\right),
$$

where $e_{3}^{*}$ is a normal vector field to $\psi^{*}(s, t) \cdot\left\{e_{1}^{*}, e_{2}^{*}, e_{3}^{*}\right\}$ is an orthonormal basis on $\psi^{*}(s, t)$. Then we obtain following theorems.

Theorem 4.8. The mean curvature $H^{*}$ of $\psi^{*}(s, t)$ is obtained as

$$
H^{*}=\frac{-\varepsilon_{2} \varepsilon_{3} \varepsilon_{5}\left(r a+2 a^{2}\left(r+\varepsilon_{2} \mu\right)-r^{2}\left(r+\varepsilon_{2} \mu\right) \kappa\right)}{\left(r+\varepsilon_{2} \mu\right)\left(2 r a+2 a^{2}\left(r+\varepsilon_{2} \mu\right)-r^{2}\left(r+\varepsilon_{2} \mu\right) \kappa\right)} .
$$


Theorem 4.9. The Gauss curvature $K^{*}$ of $\psi^{*}(s, t)$ is obtained as

$$
K^{*}=\frac{2 a^{2}-r^{2} \kappa}{\left(r+\varepsilon_{2} \mu\right)\left(2 r a+2 a^{2}\left(r+\varepsilon_{2} \mu\right)-r^{2}\left(r+\varepsilon_{2} \mu\right) \kappa\right)} .
$$

It can be easily seen that $\Psi\left(K^{*}, H^{*}\right)=K_{s}^{*} H_{t}^{*}-K_{t}^{*} H_{s}^{*}=0$. So we can give the following theorem.

Theorem 4.10. The surface $\psi^{*}(s, t)$ is a Weingarten surface.

Theorem 4.11. Let $K^{*}$ and $H^{*}$ be the Gauss curvature and mean curvature of the surface $\psi^{*}(s, t)$. Then there exists the following relation between $K^{*}$ and $H^{*}$ :

$$
-\left(r+\varepsilon_{2} \mu\right)^{2} K^{*}-2 \varepsilon_{2} \varepsilon_{3} \varepsilon_{5}\left(r+\varepsilon_{2} \mu\right) H^{*}=1
$$

where $r$ is a positive real number and $\mu$ is a real number.

From above theorem, we get the following corollary.

Corollary 4.12. The surface $\psi^{*}(s, t)$ is a linear Weingarten surface.

Definition 4.13. Let $x_{1}(\bar{s})$ be the associated curve of $x(s)$ such that $x_{1}(\bar{s})=y(s)$ where there exists a diffeomorfism $\bar{s}=f_{1}(s)$. In this paper, we will call $x_{1}(\bar{s})=y(s)$ as the first associated curve of the curve $x(s)$ in $Q^{2}$.

Let $x_{2}\left(s^{*}\right)=\alpha(s)$ where there exists a diffeomorfism $s^{*}=f_{2}(s)$ Then we will call $x_{2}\left(s^{*}\right)=\alpha(s)$ as the second associated curve of the curve $x(s)$ in $Q^{2}$.

Definition 4.14. Let $x_{1}(\bar{s})$ be the first associated curve of the curve $x(s)$ in $Q^{2}, \psi(s, t)$ and $\psi_{1}(\bar{s}, t)$ be canal surfaces (or tubular surfaces) whose center curves are $x(s)$ and $x_{1}(\bar{s})$, respectively. Then $\psi_{1}(\bar{s}, t)$ is called as "the first associated canal surface (or the first associated tubular surface)" of $\psi(s, t)$.

Similarly, let $x_{2}\left(s^{*}\right)$ be the second associated curve of the curve $x(s)$ in $Q^{2}, \psi(s, t)$ and $\psi_{2}\left(s^{*}, t\right)$ be canal surfaces (or tubular surfaces) whose center curves are $x(s)$ and $x_{2}\left(s^{*}\right)$, respectively. Then $\psi_{2}\left(s^{*}, t\right)$ is called as "the second associated canal surface (or the second associated tubular surface)" of $\psi(s, t)$.

4.1.1. The first associated curve and the first associated tubular surface. Firstly we consider the first associated curve and the first associated tubular surface of the curve $x(s)$ in $Q^{2}$. So we can give the following corollary. 
Corollary 4.15. Let $x_{1}(\bar{s})$ be the first associated curve of the curve $x(s)$ in $Q^{2}$ with the cone frame $\{x(s), \alpha(s), y(s)\}$ such that $x_{1}(\bar{s})=y(s)$ where there exists a diffeomorfism $\bar{s}=f_{1}(s)$. Then we have

$$
x_{1}=y, \quad \alpha_{1}=-\varepsilon_{1} \alpha, \quad y_{1}=x, \quad \kappa_{1}=\frac{1}{\kappa}, \quad \frac{d f_{1}}{d s}=\varepsilon_{6} \kappa,
$$

and

$$
\frac{d x_{1}}{d \bar{s}}=\alpha_{1}, \quad \frac{d \alpha_{1}}{d \bar{s}}=\kappa_{1} x_{1}-y_{1}, \quad \frac{d y_{1}}{d \bar{s}}=-\kappa_{1} \alpha_{1}
$$

where $\varepsilon_{6}=\operatorname{sgn}(\kappa),\left\{x_{1}(\bar{s}), \alpha_{1}(\bar{s}), y_{1}(\bar{s})\right\}$ is the cone frame, $\kappa_{1}(\bar{s})$ is the cone curvature of $x_{1}(\bar{s})$ and

$$
\left\langle x_{1}(\bar{s}), x_{1}(\bar{s})\right\rangle=\left\langle y_{1}(\bar{s}), y_{1}(\bar{s})\right\rangle=0, \quad\left\langle\alpha_{1}(\bar{s}), \alpha_{1}(\bar{s})\right\rangle=1, \quad\left\langle x_{1}(\bar{s}), y_{1}(\bar{s})\right\rangle=1
$$

In the following theorem, we give the parametrization of the first associated canal surfaces (or the first associated tubular surfaces) of $\psi(s, t)$.

Theorem 4.16. Let $x_{1}(\bar{s})$ be the first associated curve of the curve $x(s)$ in $Q^{2}$ with the cone frame $\{x(s), \alpha(s), y(s)\}$ such that $x_{1}(\bar{s})=y(s)$ and be the center curve of a canal surface

( $i$ ) obtained from the sphere $S_{1}^{2}(r)$. Then the parametrization of the first associated canal surface can be as following

$$
\psi_{1}(\bar{s}, t)=(1+a(\bar{s}, t)) x_{1}(\bar{s})-r(\bar{s}) r_{s}(\bar{s}) \alpha_{1}(\bar{s})+\left(\frac{r^{2}(\bar{s})\left(1-r_{\bar{s}}^{2}(\bar{s})\right)}{2 a(\bar{s}, t)}\right) y_{1}(\bar{s})
$$

and the parametrization of the first associated tubular surface can be as following

$$
\psi_{1}(\bar{s}, t)=(1+a(\bar{s}, t)) x_{1}(\bar{s})+\frac{r^{2}}{2 a(\bar{s}, t)} y_{1}(\bar{s})
$$

(ii) obtained from the hyperbolic space $H^{2}(r)$. Then the parametrization of the first associated canal surface can be as following

$$
\psi_{2}(\bar{s}, t)=(1+a(\bar{s}, t)) x_{1}(\bar{s})+r(\bar{s}) r_{\bar{s}}(\bar{s}) \alpha_{1}(\bar{s})-\left(\frac{r^{2}(\bar{s})\left(1+r_{\bar{s}}^{2}(\bar{s})\right)}{2 a(\bar{s}, t)}\right) y_{1}(\bar{s})
$$

and the parametrization of the first associated tubular surface can be as following

$$
\psi_{2}(\bar{s}, t)=(1+a(\bar{s}, t)) x_{1}(\bar{s})-\frac{r^{2}}{2 a(\bar{s}, t)} y_{1}(\bar{s})
$$

(iii) obtained from the ligthlike cone $Q^{2}$. Then the parametrization of the first associated canal surface (tubular surface) can be as following

$$
\psi_{3}(\bar{s}, t)=(1+a(\bar{s}, t)) x_{1}(\bar{s})
$$


or

$$
\psi_{4}(\bar{s}, t)=x_{1}(\bar{s})+a(\bar{s}, t) y_{1}(\bar{s})
$$

where $a(\bar{s}, t)$ is a function of $\bar{s}$ and $t$, but not only $\bar{s}$.

Firstly we consider the tubular surface $\psi_{1}(\bar{s}, t)$ for $a(\bar{s}, t)=a(t)$ as follows

$$
\begin{aligned}
\psi_{1}(\bar{s}, t) & =(1+a(t)) x_{1}(\bar{s})+\frac{r^{2}}{2 a(t)} y_{1}(\bar{s}) \\
& =\frac{r^{2}}{2 a(t)} x(s)+(1+a(t)) y(s),
\end{aligned}
$$

where $a(t)$ is nonconstant function. From (4.5), we have

$$
\begin{aligned}
\left(\psi_{1}\right)_{\bar{s}} & =\frac{\varepsilon_{6}\left(r^{2}-2 a(1+a) \kappa\right)}{2 a \kappa} \alpha, \\
\left(\psi_{1}\right)_{t} & =-\frac{r^{2} a^{\prime}}{2 a^{2}} x+a^{\prime} y
\end{aligned}
$$

which implies that

$$
\begin{aligned}
& \left\langle\left(\psi_{1}\right)_{\bar{s}},\left(\psi_{1}\right)_{\bar{s}}\right\rangle=\frac{\left(r^{2}-2 a(1+a) \kappa\right)^{2}}{4 a^{2} \kappa^{2}} \\
& \left\langle\left(\psi_{1}\right)_{\bar{s}},\left(\psi_{1}\right)_{t}\right\rangle=0, \quad\left\langle\left(\psi_{1}\right)_{t},\left(\psi_{1}\right)_{t}\right\rangle=-\frac{r^{2}\left(a^{\prime}\right)^{2}}{a^{2}} .
\end{aligned}
$$

Then

$$
\left\langle\left(\psi_{1}\right)_{\bar{s}},\left(\psi_{1}\right)_{\bar{s}}\right\rangle\left\langle\left(\psi_{1}\right)_{t},\left(\psi_{1}\right)_{t}\right\rangle-\left\langle\left(\psi_{1}\right)_{\bar{s}},\left(\psi_{1}\right)_{t}\right\rangle^{2}=-\frac{r^{2}\left(a^{\prime}\right)^{2}\left(r^{2}-2 a(1+a) \kappa\right)^{2}}{4 a^{4} \kappa^{2}}
$$

Theorem 4.17. Let $\psi_{1}(\bar{s}, t)$ be the first associated tubular surfaces of $\psi(s, t)$, which is obtained by $S_{1}^{2}(r)$. Then $\psi_{1}(\bar{s}, t)$ has a singular point at $\psi\left(s_{0}, t_{0}\right)$ if and only if

$$
r^{2}-2 a\left(t_{0}\right)\left(1+a\left(t_{0}\right)\right) \kappa\left(s_{0}\right)=0 .
$$

Now we assume that $r^{2}-2 a(t)(1+a(t)) \kappa(s) \neq 0$ for all $(t, s)$. Then we will give an orthonormal basis on $\psi_{1}(\bar{s}, t)$.

$$
\begin{aligned}
\bar{e}_{1} & =\frac{1}{\left\|\left(\psi_{1}\right)_{\bar{s}}\right\|}\left(\psi_{1}\right)_{\bar{s}}=\varepsilon_{1} \varepsilon_{2} \varepsilon_{6} \varepsilon_{7} \alpha, \\
\bar{e}_{2} & =\frac{1}{\left\|\left(\psi_{1}\right)_{t}\right\|}\left(\psi_{1}\right)_{t}=-\varepsilon_{2} \varepsilon_{3}\left(\frac{r}{2 a} x-\frac{a}{r} y\right),
\end{aligned}
$$

where $\varepsilon_{7}=\operatorname{sgn}\left(r^{2}-2 a(t)(1+a(t)) \kappa(s)\right)$ and $\left\{\bar{e}_{1}, \bar{e}_{2}\right\}$ is an orthonormal frame field on $\psi_{1}(\bar{s}, t)$. Set

$$
\bar{e}_{3}=\varepsilon_{2}\left(\frac{r}{2 a} x+\frac{a}{r} y\right),
$$


where $\bar{e}_{3}$ is a normal vector field to $\psi_{1}(\bar{s}, t) .\left\{\bar{e}_{1}, \bar{e}_{2}, \bar{e}_{3}\right\}$ is an orthonormal basis on $\psi_{1}(\bar{s}, t)$. Then similarly we obtain the following theorems.

Theorem 4.18. The mean curvature $H_{1}$ of $\psi_{1}(\bar{s}, t)$ is obtained as

$$
H_{1}=\frac{1}{2}\left(\bar{h}_{11}-\bar{h}_{22}\right)=\frac{\varepsilon_{2}\left(a \kappa+2 a^{2} \kappa-r^{2}\right)}{r\left(r^{2}-2 a \kappa-2 a^{2} \kappa\right)} .
$$

Theorem 4.19. The Gauss curvature $K_{1}$ of $\psi_{1}(\bar{s}, t)$ is obtained as

$$
K_{1}=-\left(\bar{h}_{11} \bar{h}_{22}-\left(\bar{h}_{12}\right)^{2}\right)=\frac{r^{2}-2 a^{2} \kappa}{r^{2}\left(r^{2}-2 a \kappa-2 a^{2} \kappa\right)} .
$$

Thus it can be easily seen that

$$
\Psi\left(K_{1}, H_{1}\right)=\left(K_{1}\right)_{\bar{s}}\left(H_{1}\right)_{t}-\left(K_{1}\right)_{t}\left(H_{1}\right)_{\bar{s}}=0 .
$$

So we can give the following theorems.

Theorem 4.20. The surface $\psi_{1}(\bar{s}, t)$ is a Weingarten surface.

Theorem 4.21. Let $K_{1}$ and $H_{1}$ be the Gauss curvature and mean curvature of the surface $\psi_{1}(\bar{s}, t)$. Then there exists the following relation between $K_{1}$ and $H_{1}$ :

$$
-r^{2} K_{1}-2 \varepsilon_{2} r H_{1}=1
$$

where $r$ is a positive real number.

From above theorem, we get the following corollary.

Corollary 4.22. The surface $\psi_{1}(\bar{s}, t)$ is a linear Weingarten surface.

Remark 4.23. Similar properties can be easily obtained for the tubular surface $\psi_{2}(\bar{s}, t)$.

Remark 4.24. Let $\psi_{3}(\bar{s}, t)$ and $\psi_{4}(\bar{s}, t)$ be the first associated tubular surfaces of $\psi(s, t)$, which is obtained by $Q^{2}$. Then $\psi_{3}(\bar{s}, t)$ and $\psi_{4}(\bar{s}, t)$ are degenerate surfaces.

4.1.2. The second associated curve and the second associated tubular surface. Now, we consider the second associated curve and the second associated tubular surface of the curve $x(s)$ in $Q^{2}$. So we can give the following corollaries. 
Corollary 4.25. Let $x_{2}\left(s^{*}\right)$ be the second associated curve of the curve $x(s)$ in $Q^{2}$ with $\kappa>0$ and the cone frame $\{x(s), \alpha(s), y(s)\}$ such that $x_{2}\left(s^{*}\right)=\alpha(s)$ where there exists a diffeomorfism $s^{*}=f_{2}(s)$. Then we have

$$
\begin{aligned}
& x_{2}=\alpha, \quad \alpha_{2}=\frac{1}{\sqrt{2 \kappa}}(\kappa x-y), \quad y_{2}=\frac{1}{\sqrt{2 \kappa}}(\kappa x+y), \\
& \kappa_{2}=\frac{-\kappa^{\prime}}{(2 \kappa)^{3 / 2}}, \quad \frac{d f_{2}}{d s}=\sqrt{2 \kappa},
\end{aligned}
$$

and

$$
\frac{d x_{2}}{d s^{*}}=\alpha_{2}, \quad \frac{d \alpha_{2}}{d s^{*}}=x_{2}-\kappa_{2} y_{2}, \quad \frac{d y_{2}}{d s^{*}}=-\kappa_{2} \alpha_{2}
$$

where $\left\{x_{2}\left(s^{*}\right), \alpha_{2}\left(s^{*}\right), y_{2}\left(s^{*}\right)\right\}$ is the spherical frame, $\kappa_{2}\left(s^{*}\right)$ is the spherical curvature of $x_{2}\left(s^{*}\right)$ and

$$
\left\langle x_{2}\left(s^{*}\right), x_{2}\left(s^{*}\right)\right\rangle=\left\langle y_{2}\left(s^{*}\right), y_{2}\left(s^{*}\right)\right\rangle=1, \quad\left\langle\alpha_{2}\left(s^{*}\right), \alpha_{2}\left(s^{*}\right)\right\rangle=-1 .
$$

Theorem 4.26. Let $x_{2}\left(s^{*}\right)$ be the second associated curve of the curve $x(s)$ in $Q^{2}$ with $\kappa>0$ and the cone frame $\{x(s), \alpha(s), y(s)\}$ such that $x_{2}\left(s^{*}\right)=\alpha(s)$. Also let $x_{2}\left(s^{*}\right)$ be the center curve of a canal surface

(i) obtained from the sphere $S^{2}(r)$. Then the parametrization of the second associated canal surface can be as following

$$
\begin{aligned}
\psi_{5}\left(s^{*}, t\right)= & \left(1+m_{1} r\left(s^{*}\right) \sqrt{1+r_{s^{*}}^{2}\left(s^{*}\right)} \cos t\right) x_{2}\left(s^{*}\right)+r\left(s^{*}\right) r_{s^{*}}\left(s^{*}\right) \alpha_{2}\left(s^{*}\right) \\
& +\left(m_{2} r\left(s^{*}\right) \sqrt{1+r_{s^{*}}^{2}\left(s^{*}\right)} \sin t\right) y_{2}\left(s^{*}\right)
\end{aligned}
$$

and the parametrization of the second associated tubular surface can be as following

$$
\psi_{5}\left(s^{*}, t\right)=\left(1+m_{1} r \cos t\right) x_{2}\left(s^{*}\right)+\left(m_{2} r \sin t\right) y_{2}\left(s^{*}\right)
$$

(ii) obtained from the hyperbolic space $H^{2}(r)$. Then the parametrization of the second associated canal surface can be as following

$$
\begin{aligned}
\psi_{6}\left(s^{*}, t\right)= & \left(1+m_{1} r\left(s^{*}\right) \sqrt{r_{s^{*}}^{2}\left(s^{*}\right)-1} \cos t\right) x_{2}\left(s^{*}\right)-r\left(s^{*}\right) r_{s^{*}}\left(s^{*}\right) \alpha_{2}\left(s^{*}\right) \\
& +\left(m_{2} r\left(s^{*}\right) \sqrt{r_{s^{*}}^{2}\left(s^{*}\right)-1} \sin t\right) y_{2}\left(s^{*}\right)
\end{aligned}
$$

where $m_{1}, m_{2} \in\{-1,1\}$. 
Corollary 4.27. Let $x_{2}\left(s^{*}\right)$ be the second associated curve of the curve $x(s)$ in $Q^{2}$ with $\kappa<0$ and the cone frame $\{x(s), \alpha(s), y(s)\}$ such that $x_{2}\left(s^{*}\right)=\alpha(s)$ where there exists a diffeomorfism $s^{*}=f_{2}(s)$. Then we have

$$
\begin{aligned}
& x_{2}=\alpha, \quad \alpha_{2}=\frac{1}{\sqrt{-2 \kappa}}(\kappa x-y), \quad y_{2}=\frac{1}{\sqrt{-2 \kappa}}(\kappa x+y), \\
& \kappa_{2}=\frac{-\kappa^{\prime}}{(-2 \kappa)^{3 / 2}}, \quad \frac{d f_{2}}{d s}=\sqrt{-2 \kappa},
\end{aligned}
$$

and

$$
\frac{d x_{2}}{d s^{*}}=\alpha_{2}, \quad \frac{d \alpha_{2}}{d s^{*}}=-x_{2}+\kappa_{2} y_{2}, \quad \frac{d y_{2}}{d s^{*}}=\kappa_{2} \alpha_{2}
$$

where $\left\{x_{2}\left(s^{*}\right), \alpha_{2}\left(s^{*}\right), y_{2}\left(s^{*}\right)\right\}$ is the spherical frame, $\kappa_{2}\left(s^{*}\right)$ is the spherical curvature of $x_{2}\left(s^{*}\right)$ and

$$
\left\langle x_{2}\left(s^{*}\right), x_{2}\left(s^{*}\right)\right\rangle=\left\langle\alpha_{2}\left(s^{*}\right), \alpha_{2}\left(s^{*}\right)\right\rangle=1, \quad\left\langle y_{2}\left(s^{*}\right), y_{2}\left(s^{*}\right)\right\rangle=-1 .
$$

Theorem 4.28. Let $x_{2}\left(s^{*}\right)$ be the second associated curve of the curve $x(s)$ in $Q^{2}$ with $\kappa<0$ and the cone frame $\{x(s), \alpha(s), y(s)\}$ such that $x_{2}\left(s^{*}\right)=\alpha(s)$. Also let $x_{2}\left(s^{*}\right)$ be the center curve of a canal surface

( $i)$ obtained from the sphere $S^{2}(r)$. Then the parametrization of the second associated canal surface can be as following

$$
\begin{aligned}
\psi_{7}\left(s^{*}, t\right)= & \left(1+m_{1} r\left(s^{*}\right) \sqrt{1-r_{s^{*}}^{2}\left(s^{*}\right)} \cosh t\right) x_{2}\left(s^{*}\right)-r\left(s^{*}\right) r_{s^{*}}\left(s^{*}\right) \alpha_{2}\left(s^{*}\right) \\
& +\left(m_{2} r\left(s^{*}\right) \sqrt{1-r_{s^{*}}^{2}\left(s^{*}\right)} \sinh t\right) y_{2}\left(s^{*}\right)
\end{aligned}
$$

and the parametrization of the tubular surface can be as following

$$
\psi_{7}\left(s^{*}, t\right)=\left(1+m_{1} r \cosh t\right) x_{2}\left(s^{*}\right)+\left(m_{2} r \sinh t\right) y_{2}\left(s^{*}\right)
$$

(ii) obtained from the hyperbolic space $H^{2}(r)$. Then the parametrization of the second associated canal surface can be as following

$$
\begin{aligned}
\psi_{8}\left(s^{*}, t\right)= & \left(1+m_{1} r\left(s^{*}\right) \sqrt{1+r_{s^{*}}^{2}\left(s^{*}\right)} \sinh t\right) x_{2}\left(s^{*}\right)+r\left(s^{*}\right) r_{s^{*}}\left(s^{*}\right) \alpha_{2}\left(s^{*}\right) \\
& +\left(m_{2} r\left(s^{*}\right) \sqrt{1+r_{s^{*}}^{2}\left(s^{*}\right)} \cosh t\right) y_{2}\left(s^{*}\right)
\end{aligned}
$$

and the parametrization of the tubular surface can be as following

$$
\psi_{8}\left(s^{*}, t\right)=\left(1+m_{1} r \sinh t\right) x_{2}\left(s^{*}\right)+\left(m_{2} r \cosh t\right) y_{2}\left(s^{*}\right)
$$


(iii) obtained from the ligthlike cone $Q^{2}$. Then the parametrization of the second associated canal surface(tubular surface) can be as following

$$
\psi_{9}\left(s^{*}, t\right)=\left(1+a\left(s^{*}, t\right)\right) x_{2}\left(s^{*}\right)+m_{1} a\left(s^{*}, t\right) y_{2}\left(s^{*}\right)
$$

where $m_{1}, m_{2} \in\{-1,1\}$ and $a\left(s^{*}, t\right)$ is a function of $s^{*}$ and $t$, but not only $s^{*}$.

Now, we consider the second associated tubular surface $\psi_{5}\left(s^{*}, t\right)$ of $\psi(s, t)$. Assume that $\kappa(s)=\kappa$ (constant). Let $\psi_{5}\left(s^{*}, t\right)$ be the second associated tubular surface of $\psi(s, t)$. Then we can write

$$
\begin{aligned}
\psi_{5}\left(s^{*}, t\right) & =(1+r \cos t) x_{2}\left(s^{*}\right)+(r \sin t) y_{2}\left(s^{*}\right) \\
& =\frac{\kappa r \sin t}{\sqrt{2 \kappa}} x(s)+(1+r \cos t) \alpha(s)+\frac{r \sin t}{\sqrt{2 \kappa}} y(s) .
\end{aligned}
$$

From (4.8), we have

$$
\begin{aligned}
\left(\psi_{5}\right)_{s^{*}} & =\frac{1+r \cos t}{\sqrt{2 \kappa}}(\kappa x-y) \\
\left(\psi_{5}\right)_{t} & =\frac{\kappa r \cos t}{\sqrt{2 \kappa}} x(s)-(r \sin t) \alpha(s)+\frac{r \cos t}{\sqrt{2 \kappa}} y(s),
\end{aligned}
$$

which implies that

$$
\left\langle\left(\psi_{5}\right)_{s^{*}},\left(\psi_{5}\right)_{s^{*}}\right\rangle=-(1+r \cos t)^{2}, \quad\left\langle\left(\psi_{5}\right)_{s^{*}},\left(\psi_{5}\right)_{t}\right\rangle=0, \quad\left\langle\left(\psi_{5}\right)_{t},\left(\psi_{5}\right)_{t}\right\rangle=r^{2}
$$

Then

$$
\left\langle\left(\psi_{5}\right)_{s^{*}},\left(\psi_{5}\right)_{s^{*}}\right\rangle\left\langle\left(\psi_{5}\right)_{t},\left(\psi_{5}\right)_{t}\right\rangle-\left\langle\left(\psi_{5}\right)_{s^{*}},\left(\psi_{5}\right)_{t}\right\rangle^{2}=-r^{2}(1+r \cos t)^{2}
$$

Theorem 4.29. Let $\psi_{5}\left(s^{*}, t\right)$ be the second associated tubular surfaces of $\psi(s, t)$. Then $\psi_{5}\left(s^{*}, t\right)$ has a singular point at $\psi\left(s, t_{0}\right)$ if and only if $1+r \cos t_{0}=0$.

Now we assume that $1+r \cos t \neq 0$ for all $(t, s)$. Then we will give an orthonormal basis on $\psi_{5}\left(s^{*}, t\right)$.

$$
\begin{aligned}
e_{1}^{*} & =\frac{1}{\left\|\left(\psi_{5}\right)_{s^{*}}\right\|}\left(\psi_{5}\right)_{s^{*}}=\frac{\varepsilon_{8}}{\sqrt{2 \kappa}}(\kappa x-y) \\
e_{2}^{*} & =\frac{1}{\left\|\left(\psi_{5}\right)_{t}\right\|}\left(\psi_{5}\right)_{t}=\frac{\kappa \cos t}{\sqrt{2 \kappa}} x(s)-(\sin t) \alpha(s)+\frac{\cos t}{\sqrt{2 \kappa}} y(s),
\end{aligned}
$$

where $\varepsilon_{8}=\operatorname{sgn}(1+r \cosh t)$ and $\left\{e_{1}^{*}, e_{2}^{*}\right\}$ is an orthonormal frame field on $\psi_{5}\left(s^{*}, t\right)$. Set

$$
e_{3}^{*}=\frac{\kappa \sin t}{\sqrt{2 \kappa}} x(s)+(\cos t) \alpha(s)+\frac{\sin t}{\sqrt{2 \kappa}} y(s),
$$


where $e_{3}^{*}$ is a normal vector field to $\psi_{5}\left(s^{*}, t\right) \cdot\left\{e_{1}^{*}, e_{2}^{*}, e_{3}^{*}\right\}$ is an orthonormal basis on $\psi_{5}\left(s^{*}, t\right)$. Then we obtain

$$
\begin{aligned}
D_{e_{1}^{*}} e_{1}^{*} & =\frac{1}{1+r \cos t} \alpha \\
D_{e_{1}^{*}} e_{2}^{*} & =\frac{\varepsilon_{8} \sin t}{(1+r \cos t) \sqrt{2 \kappa}}(-\kappa x+y), \\
D_{e_{2}^{*}} e_{2}^{*} & =-\frac{\kappa \sin t}{r \sqrt{2 \kappa}} x-\frac{\cos t}{r} \alpha-\frac{\sin t}{r \sqrt{2 \kappa}} y .
\end{aligned}
$$

The components of the second fundamental form $h^{*}$ are calculated as follows

$$
\begin{aligned}
& h_{11}^{*}=\left\langle D_{e_{1}^{*}} e_{1}^{*}, e_{3}^{*}\right\rangle=\frac{\cos t}{1+r \cos t}, \\
& h_{12}^{*}=\left\langle D_{e_{1}^{*}} e_{2}^{*}, e_{3}^{*}\right\rangle=0 \text { and } h_{22}^{*}=\left\langle D_{e_{2}^{*}} e_{2}^{*}, e_{3}^{*}\right\rangle=-\frac{1}{r} .
\end{aligned}
$$

Theorem 4.30. The mean curvature $\mathrm{H}_{2}$ of $\psi_{5}\left(s^{*}, t\right)$ is obtained as

$$
H_{2}=-\frac{1+2 r \cos t}{2 r(1+r \cos t)} \text {. }
$$

Theorem 4.31. The Gauss curvature $K_{2}$ of $\psi_{5}\left(s^{*}, t\right)$ is obtained as

$$
K_{2}=\frac{\cos t}{r(1+r \cos t)}
$$

From (4.9) and (4.10), we have

$$
\left(K_{2}\right)_{s^{*}}=0, \quad\left(K_{2}\right)_{t}=\frac{-\sin t}{r(1+r \cos t)^{2}}
$$

and

$$
\left(H_{2}\right)_{s^{*}}=0, \quad\left(H_{2}\right)_{t}=\frac{\sin t}{2(1+r \cos t)^{2}} .
$$

Thus it can be easily seen that $\Psi\left(K_{2}, H_{2}\right)=0$. So we can give the following theorem.

Theorem 4.32. The surface $\psi_{5}\left(s^{*}, t\right)$ is a Weingarten surface.

Also we can give the following theorem.

Theorem 4.33. Let $K_{2}$ and $H_{2}$ be the Gauss curvature and mean curvature of the surface $\psi_{5}\left(s^{*}, t\right)$. Then there exists the following relation between $K_{2}$ and $H_{2}$ :

$$
-r^{2} K_{2}-2 r H_{2}=1
$$

where $r$ is a positive real number.

From above theorem, we get the following corollary. 
Corollary 4.34. The surface $\psi_{5}\left(s^{*}, t\right)$ is a linear Weingarten surface.

Remark 4.35. Similar properties can be easily obtained for the tubular surfaces $\psi_{7}\left(s^{*}, t\right)$ and $\psi_{8}\left(s^{*}, t\right)$.

Remark 4.36. The tubular surface $\psi_{9}\left(s^{*}, t\right)$ is a degenerate surface.

4.2. Tubular surface obtained from the sphere $H^{2}(r)$. This subsection is omitted from the paper since the properties can be obtained similarly to previous subsection.

\section{REFERENCES}

[1] B.-Y. Chen, Geometry of Submanifolds, Dekker, New York, 1973.

[2] İ. Gök, Quaternionic approach of canal surfaces constructed by some new ideas, Adv. Appl. Clifford Algebras, 27 (2017), 1175-1190.

[3] M. K. Karacan, H. Es and Y. Yayll, Singular points of the tubular surfaces in Minkowski 3-space, Sarajevo J. Math. 14 (2006), 73-82.

[4] M. K. Karacan, D. W. Yoon and Y. Tuncer, Tubular surfaces of Weingarten types in Minkowski 3-space, Gen. Math. Notes, 22 (2014), 44-56.

[5] M. K. Karacan and B. Bukcu, An alternative moving frame for a tubular surface around a spacelike curve with a spacelike normal in Minkowski 3-space, Rend. Circ. Mat. Palermo, 57 (2008), 193-201.

[6] M. K. Karacan and Y. Tuncer, Tubular surfaces of Weingarten types in Galilean and pseudo-Galilean, Bull. Math. Anal. Appl. 5 (2013), 87-100

[7] E. Kocakuşaklı, O. O. Tuncer, İ. Gök and Y. Yaylı, A new representation of canal surfaces with split quaternions in Minkowski 3-space, Adv. Appl. Clifford Algebras, 27 (2017), 1387-1409.

[8] H. Liu, Curves in three dimensional Riemannian space forms, Results Math. 66 (2014), 469-480.

[9] H. Liu, Curves in the lightlike cone, Beitr. Algebra Geom. (Contribut. Algebra Geom.) 45 (2004), 291-303.

[10] J. S. Ro and D. W. Yoon, Tubes of Weingarten types in a Euclidean 3-space, J. Chungcheong Math. Soc. 22 (2009), 359-366

[11] A. Uçum and K. İlarslan, New types of canal surfaces in Minkowski 3-space, Adv. Appl. Clifford Algebras, 26 (2016), 449-468.

[12] D. W. Yoon, On non-developable ruled surfaces in Euclidean 3-spaces, Indian J. Pure Appl. Math. 38 (2007), 281-290. 